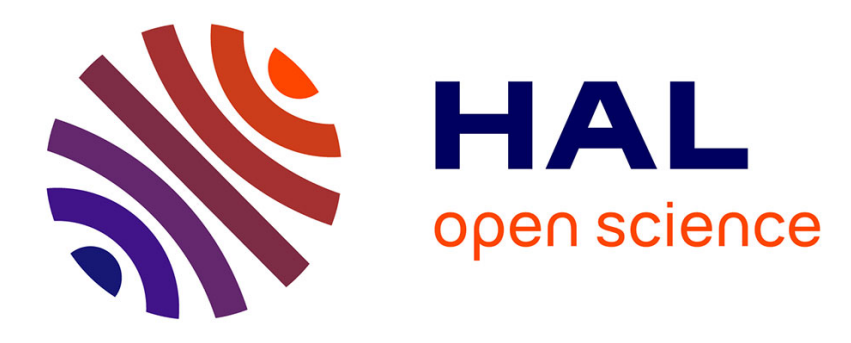

\title{
Stability estimates for some parameters of a reaction-diffusion equation coupled with an ODE
}

Muriel Boulakia, Elisa Schenone

\section{To cite this version:}

Muriel Boulakia, Elisa Schenone. Stability estimates for some parameters of a reaction-diffusion equation coupled with an ODE. Applicable Analysis, 2017, 96 (7). hal-01227427

\section{HAL Id: hal-01227427 \\ https://inria.hal.science/hal-01227427}

Submitted on 10 Nov 2015

HAL is a multi-disciplinary open access archive for the deposit and dissemination of scientific research documents, whether they are published or not. The documents may come from teaching and research institutions in France or abroad, or from public or private research centers.
L'archive ouverte pluridisciplinaire HAL, est destinée au dépôt et à la diffusion de documents scientifiques de niveau recherche, publiés ou non, émanant des établissements d'enseignement et de recherche français ou étrangers, des laboratoires publics ou privés. 


\title{
Stability estimates for some parameters of a reaction-diffusion equation coupled with an ODE
}

\author{
Muriel Boulakia* and Elisa Schenone ${ }^{\dagger}$
}

SEPTEMBER 2015

\begin{abstract}
In this work, we are interested by the monodomain equation which describes the evolution of the cardiac electrical potential and which corresponds to a coupled system involving a reaction-diffusion equation and an ordinary differential equation. We show Lipschitz stability inequalities for the identification of some parameters of the model from measurements on the cardiac potential and the ionic variable.
\end{abstract}

KEYWORDS: parameter identification, inverse problem, reaction-diffusion equation

\section{Introduction}

In this paper, we consider the monodomain model which is a coupled system involving a reactiondiffusion equation and an ordinary differential equation. The monodomain model is a classical model to describe the evolution of the electrical potential $u$ in the heart and can be directly derived from the bidomain model by assuming that the intracellular and extracellular conductivities have similar anisotropic ratio (for an introduction to the modeling in cardiac electrophysiology, we refer to [9]). In this work, we are concerned with the estimation of some parameters involved in the model from measurements on $u$ and on the ionic variable $w$

Let $\Omega$ be a bounded and regular domain in dimension 3 which corresponds to the domain occupied by the heart and let $T>0$ be a given final time. We assume that the conductivity of the cardiac medium is isotropic and homogeneous. The monodomain equations are given in $Q:=(0, T) \times \Omega$ by:

$$
\left\{\begin{array}{rlll}
\partial_{t} u-\Delta u+I_{\text {ion }}(u, w) & = & 0 & \text { in } Q \\
\partial_{t} w+f(u, w) & = & 0 & \text { in } Q .
\end{array}\right.
$$

We complete this system by the initial conditions

$$
u(0, \cdot)=u_{0} \text { in } \Omega \text { and } w(0, \cdot)=w_{0} \text { in } \Omega
$$

and by Neumann boundary conditions on $u$

$$
\nabla u \cdot n=0 \text { on }(0, T) \times \partial \Omega .
$$

*Sorbonne Universités, UPMC Univ Paris 06, CNRS, INRIA, UMR 7598, Laboratoire Jacques-Louis Lions, Equipe REO, 4, place Jussieu 75005, Paris, France

${ }^{\dagger}$ University of Luxembourg, Luxembourg 
These homogeneous Neumann conditions correspond to the assumption that the heart is electrically insulated. Monodomain equations are complemented by the choice of a ionic model which will fix the expressions of the functions $I_{\text {ion }}$ and $f$. In this work, we will assume that $I_{\text {ion }}$ and $f$ are given by:

$$
I_{\text {ion }}(u, w)=-k g(u)+r_{1}(u) w, f(u, w)=-\gamma r_{2}(u)+\beta w
$$

where $g(u)=u(u-a)(1-u)$ and $r_{1}$ and $r_{2}$ are polynomial functions given by

$$
r_{1}(u)=\chi u+1-\chi, r_{2}(u)=\varsigma u(u-a)-u, \text { where } \chi, \varsigma \in[0,1] .
$$

We could also take arbitrary polynomial functions of degree 1 for $r_{1}$ and of degree 2 for $r_{2}$ but, by this way, our writing includes the classical phenomenological models in cardiac electrophysiology like FitzHugh-Nagumo $(\chi=0, \varsigma=0)$, Alief-Panfilov $(\chi=1, \varsigma=1)$ and Roger-McCulloch $(\chi=1$, $\varsigma=0)$. We want to prove for this system stability estimates for the parameters $k$ and $\gamma$ which appear in the ionic model. To prove these estimates, we follow the Bukhgeim-Klibanov approach [2] which relies on the use of global Carleman estimates on the time derivative of the solution of the system.

We will assume that the parameters $a, k, \gamma, \beta$ only depend on space, belong to $L^{\infty}(\Omega)$ and satisfy

$$
0 \leq a(x) \leq 1, k(x) \geq k_{0}>0, \gamma(x) \geq 0, \beta(x) \geq 0, \forall x \in \bar{\Omega},
$$

and that there exists $M>0$ such that

$$
\|k\|_{L^{\infty}(\Omega)}+\|\gamma\|_{L^{\infty}(\Omega)}+\|\beta\|_{L^{\infty}(\Omega)} \leq M .
$$

Let us first give regularity results on the solution of system (1.1) completed by (1.4). These results can be proved without any major difficulty thanks to successive energy estimates on the solution and its derivatives with respect to time and thanks to elliptic energy estimates.

Proposition 1. Let $a, k, \gamma$ and $\beta$ satisfy (1.5)-(1.6).

- We assume that $\left(u_{0}, w_{0}\right) \in H^{2}(\Omega) \times L^{\infty}(\Omega)$ and that

$$
\nabla u_{0} \cdot n=0 \text { on } \partial \Omega .
$$

Then the solution of (1.1)-(1.3) completed by (1.4) satisfies

$u \in W^{1, \infty}\left(0, T ; L^{2}(\Omega)\right) \cap H^{1}\left(0, T ; H^{1}(\Omega)\right) \cap L^{\infty}\left(0, T ; H^{2}(\Omega)\right)$

$w \in W^{1, \infty}\left(0, T ; L^{\infty}(\Omega)\right)$

and

$$
\|u\|_{W^{1, \infty}\left(0, T ; L^{2}(\Omega)\right)}+\|u\|_{H^{1}\left(0, T ; H^{1}(\Omega)\right)}+\|u\|_{L^{\infty}\left(0, T ; H^{2}(\Omega)\right)}+\|w\|_{W^{1, \infty}\left(0, T ; L^{\infty}(\Omega)\right)} \leq C
$$

where $C$ depends on $\left\|u_{0}\right\|_{H^{2}(\Omega)},\left\|w_{0}\right\|_{L^{\infty}(\Omega)}, k_{0}$ and $M$.

- We assume that $\left(u_{0}, w_{0}\right) \in H^{4}(\Omega) \times H^{2}(\Omega)$ and that

$$
\nabla u_{0} \cdot n=0 \text { on } \partial \Omega \text { and } \nabla\left(\Delta u_{0}+k g\left(u_{0}\right)-r_{1}\left(u_{0}\right) w_{0}\right) \cdot n=0 \text { on } \partial \Omega \text {. }
$$

Moreover, we take $k$ and $a$ in $H^{2}(\Omega)$ and assume that there exists $\bar{M}>0$ such that

$$
\|k\|_{H^{2}(\Omega)}+\|a\|_{H^{2}(\Omega)} \leq \bar{M} .
$$

Then the solution of (1.1)-(1.3) completed by (1.4) satisfies 


$$
\begin{aligned}
& u \in W^{2, \infty}\left(0, T ; L^{2}(\Omega)\right) \cap H^{2}\left(0, T ; H^{1}(\Omega)\right) \cap W^{1, \infty}\left(0, T ; H^{2}(\Omega)\right) \\
& w \in W^{2, \infty}\left(0, T ; L^{\infty}(\Omega)\right) \\
& \text { and } \\
& \|u\|_{W^{2 . \infty}\left(0, T ; L^{2}(\Omega)\right)}+\|u\|_{H^{2}\left(0, T ; H^{1}(\Omega)\right)}+\|u\|_{W^{1, \infty}\left(0, T ; H^{2}(\Omega)\right)}+\|w\|_{W^{2, \infty}\left(0, T ; L^{\infty}(\Omega)\right)} \leq C \\
& \text { where } C \text { depends on }\left\|u_{0}\right\|_{H^{4}(\Omega)},\left\|w_{0}\right\|_{H^{2}(\Omega)}, k_{0}, M \text { and } \bar{M} .
\end{aligned}
$$

\section{Stability estimate for a reaction parameter}

Due to the writing of $f$ in (1.4), we see that we can easily get an explicit formula for the solution $w$ of the second equation in (1.1) and that the first equation of (1.1) may be viewed as a reactiondiffusion equation with a memory term. Even in the linear case, having a memory term may lead to difficulties in the theory of control and inverse problems. In [8], it is proved that the null controllability property fails for heat equations with memory. This is due to the fact that the observability inequality which is a key point to get controllability does not hold for this system.

A related problem is addressed in [3] where the null controllability of a model coupling a linear parabolic equation and an ODE is studied. If the control domain is fixed, then the controllability result does not hold and, to overcome this problem, the authors consider a control with a time-dependent support which covers the whole domain $\Omega$ and get an observability inequality for the coupled system.

Due to these remarks, it would not be possible to derive a global observability inequality for our coupled system (1.1). However, we will see that we can get stability estimates for the identification of some parameters of the model. Getting a global inequality for the monodomain equation coupled with an ODE would have allowed to prove stability estimates for more general ionic systems. In our case, we will use the fact that $w$ can be expressed explicitly with respect to $u$ and this will allow to get stability estimates for the identification of some parameters of the model.

Let us mention that paper [5] gives a simultaneous stability estimate for two parameters in the Fisher-KPP equation. For the coupling between two reaction-diffusion equations, we refer to [4] for a simultaneous stability estimate of two parameters involved in the reaction terms.

\subsection{Stability estimate for a reaction parameter in the nonlinear parabolic equation}

We are first interested by a stability estimate on the reaction parameter $k$ which appears in the first equation of (1.1). Let us consider $(\bar{u}, \bar{w})$ the solution of (1.1)-(1.4) with $k$ replaced by $\bar{k}$ in the expression of $I_{\text {ion }}$. The following theorem gives a Lipschitz stability estimate on $k-\bar{k}$ with respect to measurements on $u-\bar{u}$ and $w-\bar{w}$. In a classical way for parabolic equations, we have two types of measurements: one local measurement on the whole time interval and one global measurement at a positive time.

Theorem 1. Let us consider a nonempty open set $\omega$ included in $\Omega$. Let $(u, w)$ be the solution of (1.1), (1.3) and (1.4) with initial conditions $\left(u_{0}, w_{0}\right)$ and $(\bar{u}, \bar{w})$ be the solution of (1.1),(1.3) and (1.4) with $k$ replaced by $\bar{k}$ in the expression of $I_{i o n}$ and with initial conditions $\left(\bar{u}_{0}, \bar{w}_{0}\right)$. We assume that $\left(u_{0}, w_{0}\right) \in H^{2}(\Omega) \times L^{\infty}(\Omega)$ satisfies (1.7) and that $\left(\bar{u}_{0}, \bar{w}_{0}\right) \in H^{4}(\Omega) \times H^{2}(\Omega)$ satisfies 
(1.8).

We suppose that $a, k, \gamma, \beta$ satisfy (1.5)-(1.6), $a, \bar{k} \in H^{2}(\Omega)$ and that

$$
\|\bar{k}\|_{H^{2}(\Omega)}+\|a\|_{H^{2}(\Omega)} \leq \bar{M}
$$

for some $\bar{M}>0$. Let us assume that there exists $t_{0} \in(0, T)$ and $c_{1}>0$ such that

$$
\left|g(\bar{u})\left(t_{0}, x\right)\right| \geq c_{1}, \forall x \in \Omega .
$$

Then, there exists $C>0$ which depends on the initial conditions, $c_{1}, k_{0}, M$ and $\bar{M}$ such that

$$
\|\pi\|_{L^{2}(\Omega)} \leq C\left(\left\|p\left(t_{0}\right)\right\|_{H^{2}(\Omega)}+\left\|q\left(t_{0}\right)\right\|_{L^{2}(\Omega)}+\|p\|_{H^{1}\left(0, T ; L^{2}(\omega)\right)}\right)
$$

where $\pi=k-\bar{k}, p=u-\bar{u}$ and $q=w-\bar{w}$.

In [1], similar parameter estimates as the one in Theorem 1 were proved for the bistable equation (the first equation of $(1.1)$ with $\left.I_{\text {ion }}(u, w)=-k g(u)\right)$ thanks to a new Carleman inequality for the bistable equation. In our work, we will use the classical Carleman estimates for the heat equation [7].

Proof of Theorem 1. The difference between the systems satisfied by $(u, w)$ and $(\bar{u}, \bar{w})$ leads to

$$
\left\{\begin{aligned}
\partial_{t} p-\Delta p+k p^{3} & =k h(p, \bar{u})+\pi g(\bar{u})-\mu(p, q, \bar{u}, w) & & \text { in } Q \\
\partial_{t} q & =\gamma \varsigma p^{2}+\gamma(2 \varsigma \bar{u}-\varsigma a-1) p-\beta q & & \text { in } Q
\end{aligned}\right.
$$

where

$$
\begin{aligned}
h(p, \bar{u}) & :=(1+a-3 \bar{u}) p^{2}+\left(-a+2(1+a) \bar{u}-3 \bar{u}^{2}\right) p, \\
\mu(p, q, \bar{u}, w) & :=r_{1}(u) w-r_{1}(\bar{u}) \bar{w}=\chi \bar{u} q+\chi p w+(1-\chi) q .
\end{aligned}
$$

We see that we have an explicit formula for the solution $q$ of the second equation: for all $(t, x) \in Q$

$$
q(t, x)=q\left(t_{0}, x\right) e^{-\left(t-t_{0}\right) \beta}+\int_{t_{0}}^{t} \gamma\left[\varsigma p^{2}+(2 \varsigma \bar{u}-\varsigma a-1) p\right] e^{-\left(\tau-t_{0}\right) \beta} d \tau .
$$

Second, we consider the time derivative of the first equation of system (2.2). Then, $\rho:=\partial_{t} p$ satisfies

$$
\left\{\begin{aligned}
\partial_{t} \rho-\Delta \rho & =-3 k p^{2} \rho+k \partial_{t} h(p, \bar{u})+\pi \partial_{t} g(\bar{u})-\partial_{t} \mu(p, q, \bar{u}, w) & & \text { in } Q \\
\nabla \rho \cdot n & =0 & & \text { on }(0, T) \times \partial \Omega .
\end{aligned}\right.
$$

To simplify, we assume that $0<t_{0} \leq T / 2$ but the result still holds if $t_{0}$ is arbitrary taken in $(0, T)$ (it is sufficient to modify slightly the definition of the weight $\theta$ in what follows) and we define $T_{0}=2 t_{0} \leq T$ and $Q_{0}=\left(0, T_{0}\right) \times \Omega$.

Following [7], we can define a function $\psi$ in $\mathcal{C}^{2}(\bar{\Omega})$ such that

$$
\psi>0 \text { in } \Omega, \quad \psi=0 \text { on } \partial \Omega, \quad|\nabla \psi|>0 \text { in } \overline{\Omega \backslash \omega} .
$$

Next, we define, for all $\lambda>0$, the following weights for all $(t, x) \in Q_{0}$

$$
\varphi(t, x)=\theta(t)\left(e^{2 \lambda\|\psi\|_{L^{\infty}(\Omega)}}-e^{\lambda \psi(x)}\right) \quad \text { and } \quad \eta(t, x)=\theta(t) e^{\lambda \psi(x)}
$$


where $\theta(t)=\frac{1}{t\left(T_{0}-t\right)}$.

According to Proposition $1, u, \bar{u}$ and $w$ belong to $L^{\infty}\left(Q_{0}\right)$. Thus, if we consider the first equation of $(2.2)$ at time $t_{0}$, we obtain that

$$
\begin{array}{r}
\int_{\Omega} e^{-2 s \varphi\left(t_{0}, x\right)}|\pi(x)|^{2}\left|g(\bar{u})\left(t_{0}, x\right)\right|^{2} d x \leq \int_{\Omega} e^{-2 s \varphi\left(t_{0}, x\right)}\left|\rho\left(t_{0}, x\right)\right|^{2} d x \\
+C \int_{\Omega} e^{-2 s \varphi\left(t_{0}, x\right)}\left(\left|\Delta p\left(t_{0}, x\right)\right|^{2}+\left|p\left(t_{0}, x\right)\right|^{2}+\left|q\left(t_{0}, x\right)\right|^{2}\right) d x .
\end{array}
$$

Let us consider the first term in the right-hand side. We first notice that

$$
\begin{array}{r}
\int_{\Omega} e^{-2 s \varphi\left(t_{0}, x\right)}\left|\rho\left(t_{0}, x\right)\right|^{2} d x=\int_{0}^{t_{0}} \int_{\Omega} \partial_{t}\left(e^{-2 s \varphi}|\rho|^{2}\right) d x d t \\
\leq C \iint_{Q_{0}} e^{-2 s \varphi}\left(s^{2} \eta^{2}|\rho|^{2}+\left(s^{2} \eta\right)^{-1}\left|\partial_{t} \rho\right|^{2}\right) d x d t
\end{array}
$$

since $\left|\partial_{t} \varphi\right| \leq C|\eta|^{2}$.

According to Proposition $1, u \in L^{\infty}(Q), \quad \bar{u} \in W^{1, \infty}\left(0, T ; L^{\infty}(\Omega)\right)$ and $w \in$ $W^{1, \infty}\left(0, T ; L^{\infty}(\Omega)\right)$. Thus, if we apply the Carleman inequality for the heat equation with Neumann boundary conditions introduced in [7] and [6] to the solution $\rho$ of (2.6), we get, for $s$ and $\lambda$ large enough

$$
\begin{array}{r}
\iint_{Q_{0}} e^{-2 s \varphi}\left(s^{2} \eta^{2}|\rho|^{2}+\left(s^{2} \eta\right)^{-1}\left|\partial_{t} \rho\right|^{2}\right) d x d t \leq \frac{C}{s} \iint_{Q_{0}} e^{-2 s \varphi}|\rho|^{2} d x d t \\
+\frac{C}{s} \iint_{Q_{0}} e^{-2 s \varphi}\left(|p|^{2}+|q|^{2}+\left|\partial_{t} q\right|^{2}+|\pi|^{2}\right) d x d t+C \int_{0}^{T_{0}} \int_{\omega} e^{-2 s \varphi} s^{2} \eta^{3}|\rho|^{2} d x d t .
\end{array}
$$

Taking $s$ large enough, we can absorb the first term in the right-hand side. Next, replacing $\partial_{t} q$ by the second equation of (2.2), we get that, for $s$ and $\lambda$ large enough

$$
\begin{array}{r}
\iint_{Q_{0}} e^{-2 s \varphi}\left(s^{2} \eta^{2}|\rho|^{2}+\left(s^{2} \eta\right)^{-1}\left|\partial_{t} \rho\right|^{2}\right) d x d t \leq \frac{C}{s} \iint_{Q_{0}} e^{-2 s \varphi}|p|^{2} d x d t \\
+\frac{C}{s} \iint_{Q_{0}} e^{-2 s \varphi}|q|^{2} d x d t+\frac{C}{s} \iint_{Q_{0}} e^{-2 s \varphi}|\pi|^{2} d x d t+C \int_{0}^{T_{0}} \int_{\omega} e^{-2 s \varphi} s^{2} \eta^{3}|\rho|^{2} d x d t .
\end{array}
$$

To estimate the weighted norm of $q$ in the right-hand side of this inequality, we use (2.5) and obtain

$$
\iint_{Q_{0}} e^{-2 s \varphi}|q|^{2} d x d t \leq C \int_{\Omega} e^{-2 s \varphi\left(t_{0}\right)}\left|q\left(t_{0}, x\right)\right|^{2} d x+C \iint_{Q_{0}} e^{-2 s \varphi}|p|^{2} d x d t
$$

since $u, \bar{u} \in L^{\infty}((0, T) \times \Omega)$. By definition of $\rho=\partial_{t} p$, we have for this last term:

$$
\begin{array}{r}
\iint_{Q_{0}} e^{-2 s \varphi}|p|^{2} d x d t \leq C \iint_{Q_{0}} e^{-2 s \varphi}\left(\left|p\left(t_{0}\right)\right|^{2}+\left|\int_{t_{0}}^{t} \rho(\tau, x) d \tau\right|^{2}\right) d x d t \\
\leq C \int_{\Omega} e^{-2 s \varphi\left(t_{0}\right)}\left|p\left(t_{0}, x\right)\right|^{2} d x+C \iint_{Q_{0}} e^{-2 s \varphi}|\rho|^{2} d x d t .
\end{array}
$$


Then, combining inequalities (2.10), (2.11) and (2.12), estimate (2.9) becomes: for $s$ and $\lambda$ large enough

$$
\begin{aligned}
& \int_{\Omega} e^{-2 s \varphi\left(t_{0}, x\right)}|\pi(x)|^{2}\left|g(\bar{u})\left(t_{0}, x\right)\right|^{2} d x \leq \frac{C}{s} \int_{\Omega} e^{-2 s \varphi\left(t_{0}\right)}\left(\left|p\left(t_{0}, x\right)\right|^{2}+\left|\Delta p\left(t_{0}, x\right)\right|^{2}\right) d x \\
+ & \frac{C}{s} \iint_{Q_{0}} e^{-2 s \varphi}|\pi|^{2} d x d t+\frac{C}{s} \int_{\Omega} e^{-2 s \varphi\left(t_{0}\right)}\left|q\left(t_{0}, x\right)\right|^{2} d x+C \int_{0}^{T_{0}} \int_{\omega} e^{-2 s \varphi} s^{2} \eta^{3}|\rho|^{2} d x d t .
\end{aligned}
$$

At last, using hypothesis (2.1), we see that, for $s$ large enough, the first term in the second line can be absorbed by the left-hand side and we get the desired result.

\subsection{Stability estimate for a parameter of the ODE}

We are now interested in giving an estimation of the parameter $\gamma$ which appears in the expression of $f$ in the second equation of (1.1).

Let us call $\hat{u}, \hat{w}$ the solution of (1.1)-(1.4) with $\gamma$ replaced by $\hat{\gamma}$ in the second equation of (1.1). The following theorem gives a Lipschitz stability estimate of $\gamma-\hat{\gamma}$ with respect to measurements on $u-\hat{u}$ and $w-\hat{w}$.

Theorem 2. Let us consider a nonempty open set $\omega$ included in $\Omega$. Let $(u, w)$ be the solution of (1.1), (1.3) and (1.4) with initial conditions $\left(u_{0}, w_{0}\right)$ and $(\hat{u}, \hat{w})$ be the solution of (1.1), (1.3) and (1.4) with $\gamma$ replaced by $\hat{\gamma}$ and with initial conditions $\left(\hat{u}_{0}, \hat{w}_{0}\right)$. We assume that $\left(u_{0}, w_{0}\right) \in$ $H^{2}(\Omega) \times L^{\infty}(\Omega)$ satisfies (1.7) and that $\left(\hat{u}_{0}, \hat{w}_{0}\right) \in H^{4}(\Omega) \times H^{2}(\Omega)$ satisfies (1.8).

We suppose that $a, k, \gamma, \beta$ satisfy (1.5)-(1.6) and $a, k \in H^{2}(\Omega)$, $\|\gamma\|_{H^{2}(\Omega)} \leq \bar{M}$ for some $\bar{M}>0$. Let us assume that there exist $0<t_{0}<t_{1}<T$ and $c_{2}>0$ such that

$$
\left|r_{2}(\hat{u})(t, x)\right| \geq c_{2}, \forall(t, x) \in\left(t_{0}, t_{1}\right) \times \Omega \text { and }\left|r_{1}(\hat{u})\left(t_{1}, x\right)\right| \geq c_{2}, \forall x \in \Omega .
$$

Then, there exists $C>0$ which depends on the initial conditions, $c_{2}, k_{0}, M$ and $\bar{M}$ such that

$$
\|\alpha\|_{L^{2}(\Omega)} \leq C\left(\left\|z\left(t_{1}\right)\right\|_{H^{2}(\Omega)}+\left\|v\left(t_{0}\right)\right\|_{L^{2}(\Omega)}+\|z\|_{H^{1}\left(0, T ; L^{2}(\omega)\right)}\right)
$$

where $\alpha=\gamma-\hat{\gamma}, z=u-\hat{u}$ and $v=w-\hat{w}$.

Proof of Theorem 2. The difference between the systems satisfied by $(u, w)$ and $(\hat{u}, \hat{w})$ leads to

$$
\left\{\begin{aligned}
\partial_{t} z-\Delta z+k z^{3} & =k h(z, \hat{u})-\mu(z, v, \hat{u}, w) \\
\partial_{t} v & =\gamma\left(\varsigma z^{2}+2 \varsigma \hat{u} z-(\varsigma a+1) z\right)-\alpha\left(\varsigma \hat{u}^{2}-(\varsigma a+1) \hat{u}\right)-\beta v
\end{aligned}\right.
$$

where $h(z, \hat{u})$ and $\mu(z, v, \hat{u}, w)$ are defined in (2.3)-(2.4). As in the proof of Theorem 1, the solution $v$ of the second equation can be expressed with an explicit formula: for all $(t, x) \in Q$

$$
v(t, x)=v\left(t_{0}, x\right) e^{-\left(t-t_{0}\right) \beta}+\int_{t_{0}}^{t}\left[\gamma\left(\varsigma z^{2}+2 \varsigma \hat{u} z-(\varsigma a+1) z\right)-\alpha r_{2}(\hat{u})\right] e^{-\left(\tau-t_{0}\right) \beta} d \tau .
$$

First, we consider the time derivative of the first equation of system (2.14)

$$
\left\{\begin{aligned}
\partial_{t} \zeta-\Delta \zeta & =-3 k z^{2} \zeta+k \partial_{t} h(z, \hat{u})-\partial_{t} \mu(z, v, \hat{u}, w) & & \text { in } Q \\
\nabla \zeta \cdot n & =0 & & \text { on }(0, T) \times \partial \Omega
\end{aligned}\right.
$$


where $\zeta=\partial_{t} z$.

As in the proof of Theorem 1, we assume, without loss of generality that $0<t_{0}+t_{1} \leq T$ and we define $T_{0}=t_{0}+t_{1} \leq T$ and $Q_{0}=\left(0, T_{0}\right) \times \Omega$.

Let us now introduce a function $\psi$ in $\mathcal{C}^{2}(\bar{\Omega})$ which satisfies (2.7) and consider the functions $\varphi$ and $\eta$ defined in (2.8), with a new function $\theta \in \mathcal{C}^{\infty}\left(0, T_{0}\right)$ which satisfies

$$
\theta(t)= \begin{cases}\frac{1}{t\left(T_{0}-t\right)}, & 0<t \leq \frac{2 t_{0}}{3} \\ \text { strictly decreasing, } & \frac{2 t_{0}}{3}<t \leq t_{0} \\ \text { constant }, & t_{0}<t \leq t_{1} \\ \theta\left(T_{0}-t\right), & t_{1}<t \leq T_{0} .\end{cases}
$$

Let us consider the first equation of (2.14) and replace $\mu(z, v, \hat{u}, w)$ by its definition (2.4). We get that

$$
r_{1}(\hat{u}) v=-\chi z w-\zeta+\Delta z-k z^{3}+k h(z, \hat{u}) .
$$

Next, we take this equation at time $t_{1}$ and replace $v\left(t_{1}\right)$ thanks to formula (2.15). Thanks to Proposition $1, w, u$ and $\hat{u}$ belong to $L^{\infty}((0, T) \times \Omega)$ and we deduce that, for all $x \in \Omega$

$$
\begin{aligned}
& \left|\alpha(x) r_{1}(\hat{u})\left(t_{1}, x\right) \int_{t_{0}}^{t_{1}} r_{2}(\hat{u})(t, x) e^{-\left(t-t_{0}\right) \beta} d t\right| \\
& \leq C\left(\left|z\left(t_{1}, x\right)\right|+\left|\zeta\left(t_{1}, x\right)\right|+\left|\Delta z\left(t_{1}, x\right)\right|+\left|v\left(t_{0}, x\right)\right|+\int_{t_{0}}^{t_{1}}|z(t, x)| d t\right)
\end{aligned}
$$

We take the square of this expression, multiply by $e^{-2 s \varphi\left(t_{1}\right)}$ and integrate over $\Omega$, we get

$$
\begin{aligned}
& \int_{\Omega} e^{-2 s \varphi\left(t_{1}, x\right)}|\alpha(x)|^{2}\left|r_{1}(\hat{u})\left(t_{1}, x\right)\right|^{2}\left|\int_{t_{0}}^{t_{1}} r_{2}(\hat{u})(t, x) e^{-\left(t-t_{0}\right) \beta} d t\right|^{2} d x \\
& \leq C\left(\left\|z\left(t_{1}\right)\right\|_{H^{2}(\Omega)}^{2}+\left\|v\left(t_{0}\right)\right\|_{L^{2}(\Omega)}^{2}+\int_{\Omega} e^{-2 s \varphi\left(t_{1}\right)}\left|\zeta\left(t_{1}\right)\right|^{2} d x+\iint_{Q_{0}} e^{-2 s \varphi}|z|^{2} d x d t\right) .
\end{aligned}
$$

For the third term in the right-hand side, we have

$$
\begin{aligned}
& \int_{\Omega} e^{-2 s \varphi\left(t_{1}\right)}\left|\zeta\left(t_{1}\right)\right|^{2} d x=\int_{0}^{t_{1}} \int_{\Omega} \partial_{t}\left(e^{-2 s \varphi}|\zeta|^{2}\right) d x d t \\
& \leq C \iint_{Q_{0}} e^{-2 s \varphi}\left(s^{2} \eta^{2}|\zeta|^{2}+\left(s^{2} \eta\right)^{-1}\left|\partial_{t} \zeta\right|^{2}\right) d x d t .
\end{aligned}
$$

According to Proposition $1, u \in L^{\infty}(Q), \hat{u} \in W^{1, \infty}\left(0, T ; L^{\infty}(\Omega)\right)$ and $w \in W^{1, \infty}\left(0, T ; L^{\infty}(\Omega)\right)$. Thanks to the Carleman inequality for parabolic equations with Neumann boundary conditions ([7] and [6]) applied to $\zeta$ solution of (2.16), we get, for $s$ and $\lambda$ large enough

$$
\begin{aligned}
& \iint_{Q_{0}} e^{-2 s \varphi}\left(s^{2} \eta^{2}|\zeta|^{2}+\left(s^{2} \eta\right)^{-1}\left|\partial_{t} \zeta\right|^{2}\right) d x d t \\
& \leq \frac{C}{s} \iint_{Q_{0}} e^{-2 s \varphi}\left(|z|^{2}+|v|^{2}+\left|\partial_{t} v\right|^{2}\right) d x d t+C \int_{0}^{T_{0}} \int_{\omega} e^{-2 s \varphi} s^{2} \eta^{3}|\zeta|^{2} d x d t .
\end{aligned}
$$

We then argue as in estimates (2.12) for the integral in $v$ in the right-hand side and replace $\partial_{t} v$ by the second equation of (2.14) and thus, the last two terms of (2.18) become, for $s$ and $\lambda$ large 
enough

$$
\begin{aligned}
& \int_{\Omega} e^{-2 s \varphi\left(t_{1}\right)}\left|\zeta\left(t_{1}\right)\right|^{2} d x+\iint_{Q_{0}} e^{-2 s \varphi}|z|^{2} d x d t \\
& \leq C\left\|v\left(t_{0}\right)\right\|_{L^{2}(\Omega)}^{2}+\frac{C}{s} \int_{\Omega} e^{-2 s \varphi\left(t_{0}, x\right)}|\alpha(x)|^{2} d x+C \int_{0}^{T_{0}} \int_{\omega} e^{-2 s \varphi} s^{2} \eta^{3}|\zeta|^{2} d x d t .
\end{aligned}
$$

Putting this inequality in (2.18), we get, for $s$ and $\lambda$ large enough

$$
\begin{aligned}
& \int_{\Omega} e^{-2 s \varphi\left(t_{1}, x\right)}|\alpha(x)|^{2}\left|r_{1}(\hat{u})\left(t_{1}, x\right)\right|^{2}\left|\int_{t_{0}}^{t_{1}} r_{2}(\hat{u})(t, x) e^{-\left(t-t_{0}\right) \beta} d t\right|^{2} d x \\
& \leq C\left(\left\|z\left(t_{1}\right)\right\|_{H^{2}(\Omega)}^{2}+\left\|v\left(t_{0}\right)\right\|_{L^{2}(\Omega)}^{2}++\frac{C}{s} \int_{\Omega} e^{-2 s \varphi\left(t_{0}, x\right)}|\alpha(x)|^{2} d x+C \int_{0}^{T_{0}} \int_{\omega} e^{-2 s \varphi} s^{2} \eta^{3}\left|\partial_{t} z\right|^{2} d x d t\right) .
\end{aligned}
$$

Using hypothesis (2.13), we conclude the proof.

\section{Bibliography}

[1] M. Boulakia, C. Grandmont, and A. Osses. Some inverse stability results for the bistable reaction-diffusion equation using carleman inequalities. C.R. Math. Acad. Sci. Paris, Ser I, 347(11-12):619-622, 2009.

[2] A. Bukhgeim and M. Klibanov. Global uniqueness of a class of inverse problems. Sov. Math. Dokl., 24:244-247, 1982.

[3] F. W. Chaves-Silva, L. Rosier, and E. Zuazua. Null controllability of a system of viscoelasticity with a moving control. Journal de Mathématiques Pures et Appliquées, 1(2):198-222, 2014.

[4] M. Cristofol, P. Gaitan, H. Ramoul, and M. Yamamoto. Identification of two coefficients with data of one component for a nonlinear parabolic system. Appl. Anal., 91(11):2073-2081, 2012.

[5] M. Cristofol and L. Roques. Stable estimation of two coefficients in a nonlinear Fisher-KPP equation. Inverse Problems, 29(9):095007, 18, 2013.

[6] E. Fernández-Cara, M. González-Burgos, S. Guerrero, and J.-P. Puel. Null controllability of the heat equation with boundary Fourier conditions: the linear case. ESAIM Control Optim. Calc. Var., 12(3):442-465, 2006.

[7] A.V. Fursikov and O.Yu. Imanuvilov. Controllability of evolution equations. Volume 34 of Lecture Notes Series. Seoul National University Research Institute of Mathematics Global Analysis Research Center, Seoul, 1996.

[8] S. Guerrero and O.Yu. Imanuvilov. Remarks on non controllability of the heat equation with memory. ESAIM: Control, Optimisation and Calculus of Variations, 19:288-300, 2013.

[9] J. Sundnes, G.T. Lines, X. Cai, B.F. Nielsen, K.-A. Mardal, and A. Tveito. Computing the electrical activity in the heart, volume 1 of Monographs in Computational Science and Engineering. Springer-Verlag, Berlin, 2006. 